\title{
Essais de germination et suivi des performances de croissance des plants de Khaya senegalensis (Desv.) A. Juss., en zone soudanienne (Côte d'Ivoire)
}

Pagadjovongo Adama SILUE ${ }^{(1)^{*}}$, Kouadio Arsène Dieudonné KOFFI ${ }^{(2)}$ Adjoua Bénédicte KOFFI $^{(1)}$ Konan Edouard KOUASSI ${ }^{(2)}$

(1) Département de Biologie Végétale, UFR Sciences Biologiques, Université Péléforo Gbon Coulibaly, BP 1328 Korhogo (Côte d'Ivoire)

(2) Laboratoire de Milieux Naturels et Conservation de la Biodiversité, UFR Biosciences, Université Félix HouphouëtBoigny 22 BP 582 Abidjan 22 (Côte d'Ivoire).

*Auteur correspondant; E-mail : pagadsilue@gamail.com ; Tel. : (+225) 0707791431

Mots clés : Qualités germinatives, propagation, profondeur de semis, stumps, production de plants.

Keywords: Germination qualities, spread, sowing depth, stumps, seedling production.

Date of Acceptance 14/04/2021, Publication date 31/05/2021, http://m.elewa.org/Journals/about-japs/

\section{RESUME}

Khaya senegalensis (Acajou de savane) est une espèce de bois d'œuvre couramment exploitée abusivement dans les zones soudaniennes de la Côte d'Ivoire. Cette étude, visant à améliorer la domestication de l'espèce, a testé deux modes de multiplication, l'une utilisant directement les graines et l'autre des portions de tiges pourvues de système racinaire appelées « stumps ». Le matériel de propagation a été collecté sur des sujets de l'espèce puis soumis à l'influence de facteurs abiotiques (intensité de l'ensoleillement, profondeur de semis, types de sols). Les résultats ont montré que les graines traitées à l'eau présentent de bonnes qualités germinatives avec des taux de germination de l'ordre de $80 \%$, une énergie germinative d'au moins $50 \%$ et une valeur culturale pouvant atteindre $80 \%$, que cela soit en milieu ombragé ou milieu ensoleillé. Concernant la variation de la profondeur de semis sur les paramètres de germination, les graines de $K$. senegalensis produisent les meilleurs résultats de germination pour des profondeurs de semis de 1 à $3 \mathrm{~cm}$. La croissance des plants issus de stumps atteint une hauteur de $35 \mathrm{~cm}$ contre $28 \mathrm{~cm}$ pour les plants issus de graines, en milieu ombragé. Ainsi, la reproduction par voie de stumps présente une technique supplémentaire à la production de plants en pépinière pour le reboisement de l'espèce.

\begin{abstract}
Khaya senegalensis (Savannah mahogany) is a timber species commonly exploited nowadays in the Sudanese areas of Côte d'Ivoire._This study aimed at improving the domestication of the species tested two modes of propagation, one using the seeds directly and the other portions of stems provided with a root system called "stumps". Propagation material was collected from subjects of the species and then exposed to the influence of abiotic factors (intensity of sunshine, depth of sowing, types of soil). The results have shown that the seeds treated with water have good germination qualities with germination rates of the order of 80 $\%$, a germination energy of at least $50 \%$ and a cultural value of up to $80 \%$, than either in shaded or sunny surroundings. Regarding the variation of the sowing depth on the germination parameters, the seeds of Khaya senegalensis produce the best germination results for sowing depths of 1 to $3 \mathrm{~cm}$. The growth of the plants resulting from stumps reaches
\end{abstract}


a height of $35 \mathrm{~cm}$ against $28 \mathrm{~cm}$ for the plants resulting from seeds, in shaded environment. So, reproduction by means of stumps presents an additional technique to the production of seedlings in the nursery for the reforestation of the species.

\section{INTRODUCTION}

Le souci de conservation de la biodiversité, avec la prise en compte des besoins et aspirations des populations locales, est devenu réel depuis le Sommet de la Terre en 1992 à Rio (Inoussa et al., 2013). En Afrique de l'Ouest, les espèces végétales ligneuses procurent à l'homme des revenus, des produits comestibles, de médecine traditionnelle, d'énergie, et d'autres aspects du bien-être humain (Olivier et al., 2012 ; Sanogo et al., 2013 ; Douma et al., 2019). Aussi, les ligneux et leurs habitats subissent des perturbations, liées à ces actions anthropiques et aux changements climatiques, qui menacent leur survie. En Côte d'Ivoire, la pratique intense de l'exploitation forestière entraine aujourd'hui la dégradation de plusieurs forêts et habitats naturels ainsi que la raréfaction de nombreuses espèces (FAO, 2005). Parmi les espèces couramment exploitées et menacées de disparition dans le Nord de la Côte d'Ivoire, figure Khaya senegalensis qui fait l'objet de cette étude. C'est une espèce à usages multiples et l'un des plus grands arbres et des plus majestueux parmi ceux existant dans la Région soudanienne (Eyog, 1987). Divers organes de l'espèce sont utilisés pour leurs propriétés médicinales (Kolawole et al., 2012). Les feuilles sont utilisées comme fourrage en zone soudanosahélienne et le bois comme matériau de construction, pour la fabrication de pirogues, des ustensiles de ménage (Silué, 2018). C'est donc un arbre agroforestier qui constitue une source de

\section{MATERIEL ET METHODES}

3.1. Zone d'étude: Les travaux ont été conduits de février à octobre 2016, dans la pépinière du Cantonnement des Eaux et Forêts du Département de Kouto (Figure 1). Le site est à la latitude $9^{\circ} 52$ '50" nord, et à la longitude 6²4'14" ouest, sur une altitude de $363 \mathrm{~m}$. Le climat est du type soudanien et est caractérisé par l'alternance d'une saison sèche (novembre à mai) revenu très importante pour les populations mais qui se raréfie de plus en plus dans les différents écosystèmes soudaniens. Dans le nord-ouest de la Côte d'Ivoire, l'exploitation abusive (sciage, émondage) de $K$. senegalensis constitue un frein à la production des semences et compromet ainsi la régénération naturelle qui augmente les risques de propension à la régression de l'espèce. Les différentes pressions anthropiques sur $K$. senegalensis combinées aux difficultés de régénération lui confèrent le statut d'espèce vulnérable menacée d'extinction sur la liste rouge de l'UICN (Adjahossou et al., 2018). Par ailleurs, la longévité des graines à la température ambiante est de l'ordre de 6 à 8 mois ; elles perdent ensuite assez rapidement leur faculté germinative (CIRAD, 1988). La survie de cette espèce, désormais, repose sur des approches de gestion appropriées associées à des techniques de régénération artificielle. La présente étude sur les qualités de germination des graines et la croissance juvénile des plants a été entreprise afin d'approfondir et renforcer les connaissances sur la domestication de l'espèce et de contribuer à sa protection et sa conservation durable. De manière spécifique, il s'agit de tester et de comparer, sous l'influence de certains facteurs abiotiques, deux techniques de multiplication artificielle de l'espèce, à savoir le semis directe avec des graines et le repiquage de stumps.

à une saison pluvieuse (juin à octobre), avec un indice pluviométrique compris entre 1000 et $1400 \mathrm{~mm}$. Les températures moyennes mensuelles sont comprises entre $25^{\circ} \mathrm{C}$ et $31^{\circ} \mathrm{C}$. Les sols comprennent, les lithosols (4\%), les vertisols $(39 \%)$ et les sols ferrugineux $(57 \%)$ selon Beaudou et Sayol (1980). 

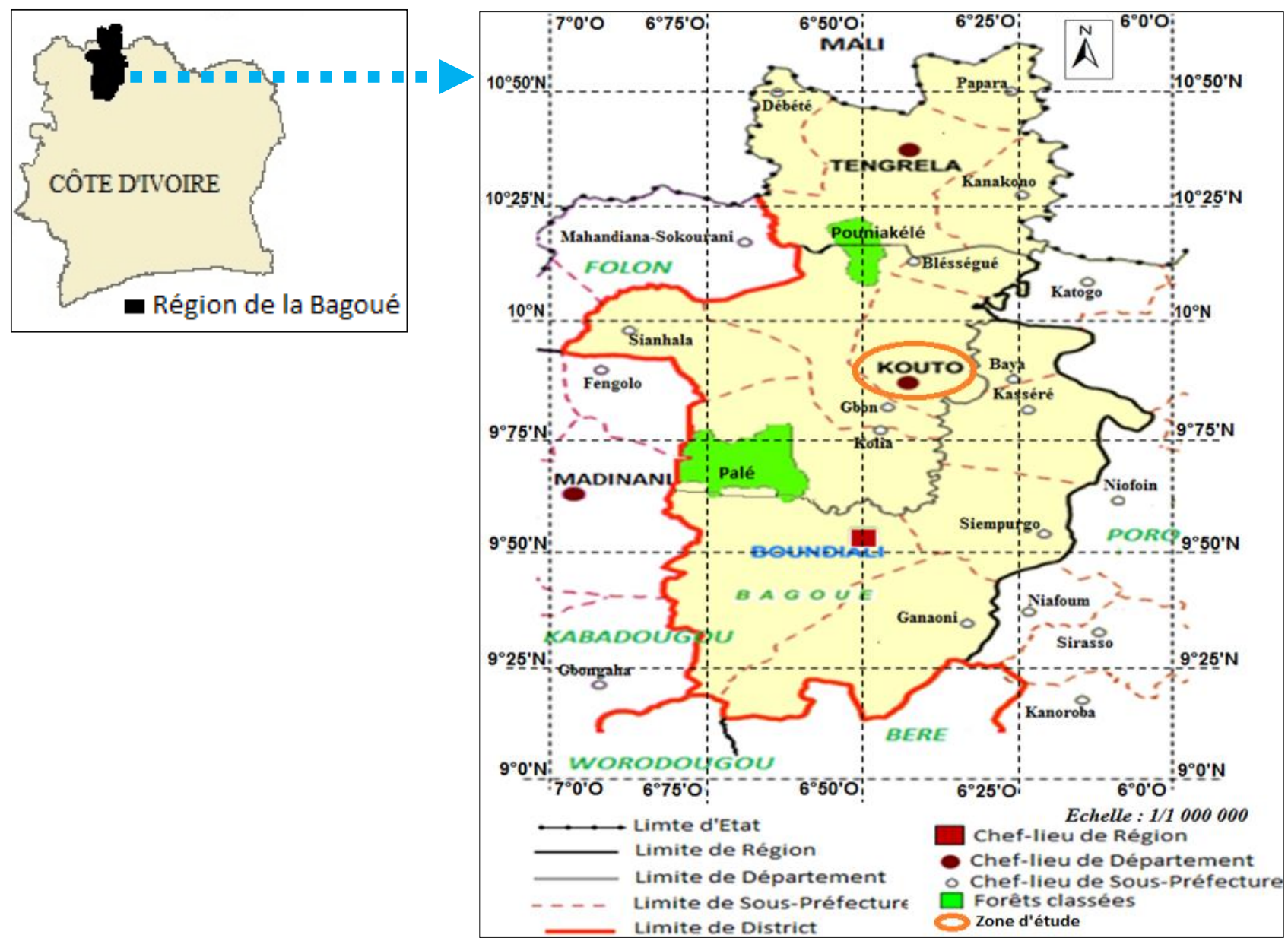

Figure 1: Carte de localisation de la zone d'étude

3.2. Matériel végétal: Le matériel végétal provient de l'espèce $K$. senegalensis. Les graines triées servent de semences tandis que les stumps constituent le matériel de base de la multiplication végétative (Figure 2). Les semences utilisées, pour tous les essais expérimentaux, ont été récoltées dans les terroirs villageois, sur des semenciers identifiés (au total 5 semenciers) pour leur forte productivité et leur qualité de graines. Les stumps sont des portions $(15-20 \mathrm{~cm})$ de plants dont la partie aérienne a été supprimée au-dessus des premiers bourgeons et prélevés sur une pépinière réalisée un an plutôt. 


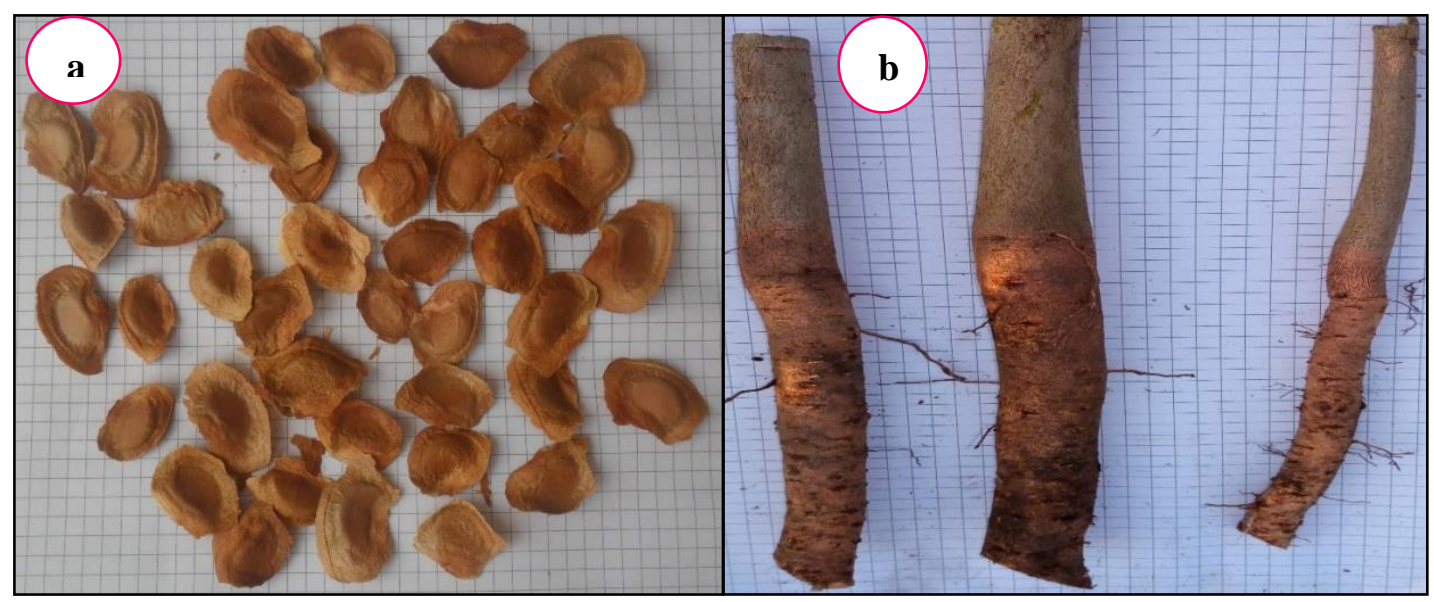

Figure 2 : Types de matériel végétal de Khaya senegalensis, (a) graines, (b) stumps

\subsection{Dispositif expérimental et collecte} des données : Les essais de germination ont été effectués sous l'effet de trois (3) facteurs abiotiques. Il s'agit de l'intensité de l'ensoleillement, la profondeur de semis et des types de sols. L'intensité de l'ensoleillement a été mise en évidence par un dispositif composant deux milieux : un milieu fortement ensoleillé ou milieu ouvert et un milieu faiblement ensoleillement matérialisé par une ombrière constitué de claie fortement recouverte de feuilles de palmier à huile. Pour cet essai, un prétraitement à l'eau froide a été effectué pendant 48 heures sur un lot de semences. L'influence de la profondeur de semis sur les paramètres de germination a été mise en évidence par des semis effectués à différents niveaux de profondeur : P1 $(1 \mathrm{~cm})$; P2 $(2 \mathrm{~cm})$; P3 $(3 \mathrm{~cm})$; P5 $(5 \mathrm{~cm})$; P7 $(7 \mathrm{~cm})$ et P9 $(9 \mathrm{~cm})$. Pour l'étude de la variation du type de sol sur les qualités germinatives des semences, les essais ont été effectués sur les sols sableux, argileux et gravillonnaire. Ces sols correspondent aux principaux sols rencontrés dans la zone d'étude. Les essais de germination ont débuté en février 2016 et se sont effectués sur une période de 32 semaines en conditions naturelles d'éclairement, de température et d'hygrométrie. Les essais ont été conduits selon un dispositif constitué de cinq blocs (ou répétition) de 100 sachets ou unités expérimentales (Figure 3). Les semis ont été effectués dans des sachets en film plastique polyéthylène de $18 \mathrm{~cm}$ de hauteur pour $10 \mathrm{~cm}$ de diamètre. Une graine est semée au centre des sachets dans un poquet profond de $1 \mathrm{~cm}$ environ, après leur arrosage jusqu'à ce que le substrat soit à sa capacité au champ une heure avant. Par la suite, deux arrosages plus légers ont lieu chaque jour, le matin ( 7 à 8 h) et l'après-midi (16 à 17 h). Le comptage des graines germées a été effectué tous les jours pendant deux semaines et celui des graines saines non germées en fin d'expérience, afin d'évaluer et d'exprimer les paramètres de germinations. L'étude de la croissance des plants de K. senegalensis a consisté à mesurer la hauteur (croissance verticale) comprise entre le collet et le bourgeon terminal et le diamètre au collet (croissance horizontale) de 15 individus de plants issus de la germination de graines et 15 individus de plants issus de stumps, choisis de façon aléatoire dans des lots de 100 individus de chaque catégorie de matériel végétal. Les mensurations se sont effectuées sur une période de cinq mois en milieu ombragé et en milieu ouvert. 


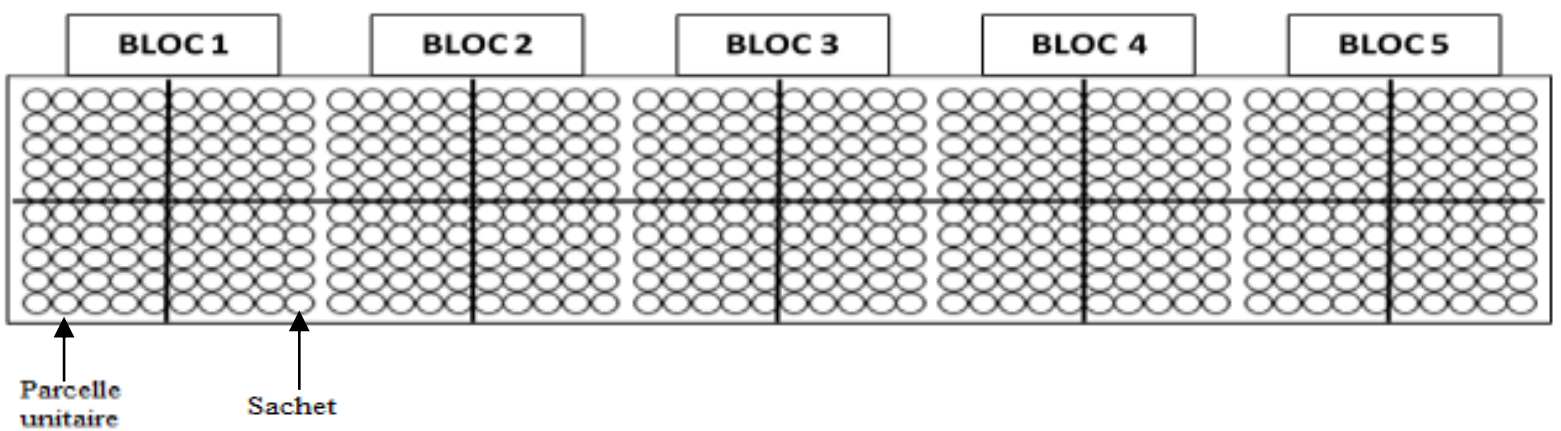

Figure 3: Schéma du dispositif expérimental de semis des graines des trois espèces étudiées en pépinière

\subsection{Méthodes d'expression et d'analyse} statistique des données: La comparaison portera sur les paramètres suivants :

- le Temps de Latence (TL) qui fait référence au temps écoulé de la date de semis à l'apparition des premières germinations ;

- $\quad$ le Taux de Germination qui est égal au rapport du nombre de graines germées (TG) à celui du nombre de graines semées $(\mathrm{N})$, multiplié par 100 ;

- l'Énergie Germinative (EG) qui est le pourcentage de semences (en nombre) qui germent jusqu'au moment de germination maximale, correspondant généralement à la période de 24 heures où se produit le plus grand nombre de germination;

- la Valeur Culturale (VC) qui est l'expression de la combinaison du taux de germination (TG) et du taux de pureté (TP), qui

\section{RESULTATS}

4.1. Influence de l'intensité de l'ensoleillement sur les qualités germinatives des semences: Le tableau 1 présente les valeurs des paramètres de germination dans chacun des milieux. De l'analyse de ces données, les semences traitées ont un temps de latence relativement plus court (13 jours environ) quel que soit le milieu. Pour le taux de germination, on constate systématiquement que les valeurs moyennes les plus élevées $(86,4 \pm 3,91 \%$ à $81,8 \pm 10,26 \%)$ sont atteintes dans le milieu ombragé. Ces valeurs montrent que le milieu ombragé favorise est la somme des semences germées et celles susceptibles de germer divisée par 100 ;

- la Vitesse de Croissance (VCr) en hauteur des plants qui est la proportion de croissance maximale par unité de temps au cours d'une période donnée.

Les moyennes des paramètres de germination et de croissance ont été comparées entre elles à l'aide du test $\mathrm{t}$ de Student (cas de normalité) et celui de Mann-Withney (absence de normalité). Ces deux tests ont été appliqués en présence de deux échantillons. En présence de plus de deux échantillons, le test ANOVA (cas de normalité) a été utilisé et celui de Kruskal-Wallis (absence de normalité) a été appliqué. Dans le cadre de l'étude de la variation de la profondeur de semis, où plusieurs paramètres à caractères qualitatifs et quantitatifs ont été pris en compte, l'Analyse Factorielle Multiple (AFM) a été la méthode la mieux indiquée.

la germination des semences de $K$. senegalensis. Pour les deux autres paramètres que sont l'énergie germinative et la valeur culturale, les forts taux moyens sont enregistrés dans le milieu ombragé, quel que soit la semence, avec des valeurs respectives de $53 \pm 9,43 \%$ et $80,95 \pm$ $5,99 \%$

4.2. Influence de la profondeur de semis sur les qualités germinatives des semences : La figure 4 présente la carte factorielle de l'Analyse Factorielle Multiple relative à l'influence de la profondeur de semis sur les paramètres de germination des semences de $K$. 
senegalensis. L'examen du premier plan factoriel des variables, met en évidence un axe 1 qui décrit, du côté positif, les profondeurs de semis P1, P2, P3 et P5. Dans sa partie négative, l'on a les profondeurs de semis P7 et P9. L'axe 2 décrit, du côté positif, les profondeurs P2, P5 et P7 et, du côté négatif, les profondeurs P1, P3 et P9. Selon ses axes, ces quatre groupes de profondeurs sont distincts en se basant sur les paramètres de germination. Le premier groupe est $\mathrm{P}$ 9, le second est $\mathrm{P} 7$, le troisième groupe est $\mathrm{P} 5$ et enfin le quatrième groupe est $\mathrm{P} 1, \mathrm{P} 2$ et $\mathrm{P} 3$. Les valeurs moyennes des paramètres de germination pour chaque groupe sont données par le tableau 2. Le temps de latence est relativement court pour le groupe 4 avec une valeur moyenne de 13,06 \pm 0,59 jours. Ce délai de germination est pratiquement le double pour le groupe 1. En considérant le taux de germination, la valeur moyenne la plus élevée, $83,06 \pm 4,79 \%$, est obtenue avec le groupe 4 . La valeur moyenne, pour ce paramètre, est plus faible, $17,4 \pm 1,14 \%$, pour le groupe 1 . S'agissant de l'énergie germinative, le groupe 4 enregistre le taux le plus élevé, $52,87 \pm 7,62 \%$ Ce taux est très faible pour le groupe 1. Quant à la valeur culturale, le groupe 4 se distingue avec une valeur moyenne de 72,14 $\pm 7,14 \%$ La valeur de ce paramètre est très faible, $3,64 \pm 0,48 \%$, pour le groupe 1.

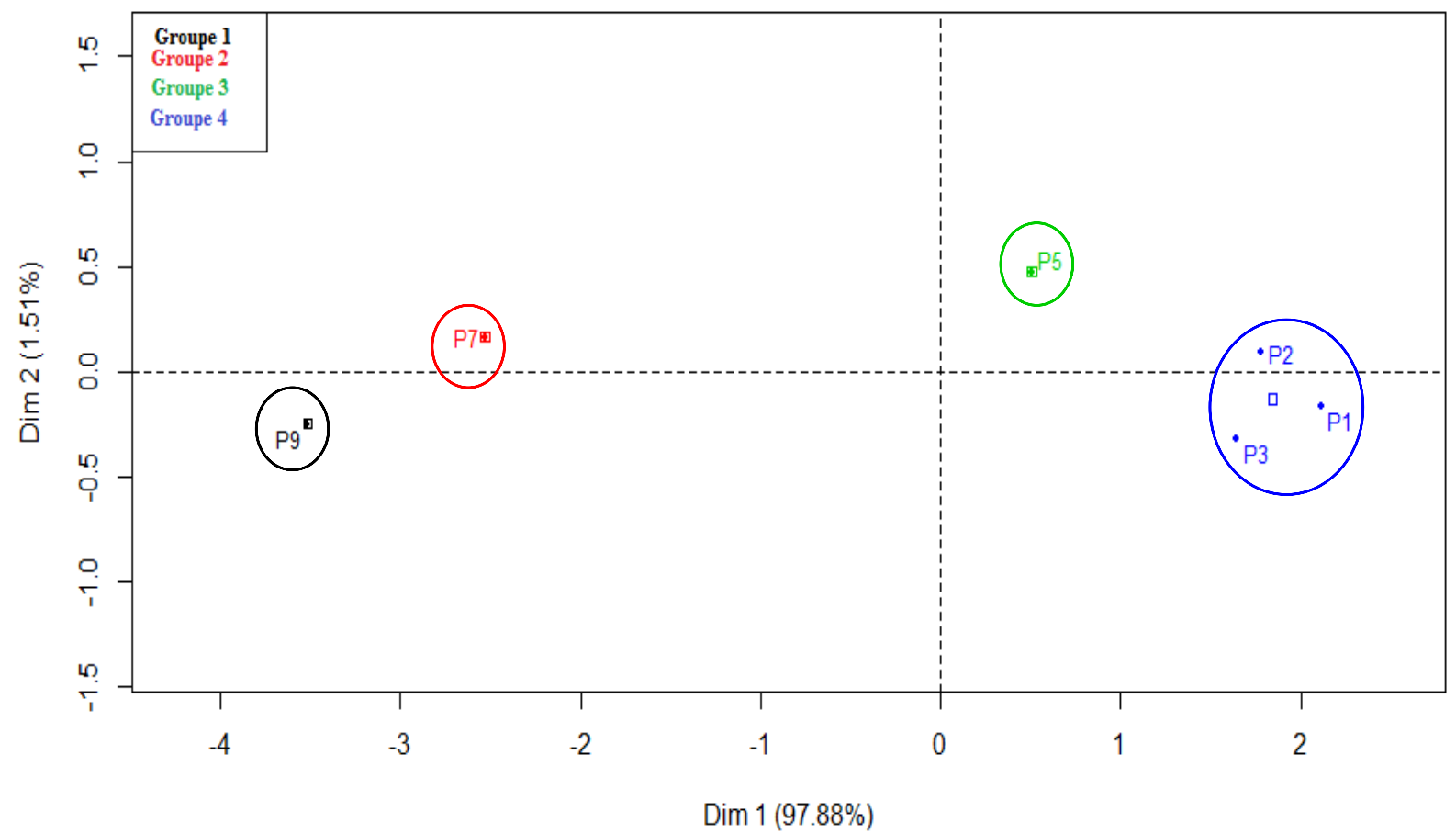

Figure 4 : Carte factorielle de l'effet des profondeurs de semis sur les paramètres de germination des semences de Khaya senegalensis

4.3. Influence des types de sols sur les qualités germinatives des semences: Le tableau 3 présente les résultats culturaux obtenus sur les différents sols. Les sols sableux et argileux présentent pratiquement le même temps de latence (12,6 \pm 0,55 jours). Le sol gravillonnaire présente un temps de latence moyen relativement plus élevé (15,6 \pm 3,8 jours). Mais les différences entres les valeurs moyennes ne sont pas significatives. Pour le taux de germination, le sol sableux induit de meilleures possibilités de germination $(61,8 \pm 4,6 \%)$ par rapport aux deux autres sols, avec respectivement $30,9 \pm 6,1 \%$ et $16 \pm 3,53$ p. c. pour les sols argileux et gravillonnaire. En ce qui concerne l'énergie germinative, le sol sableux enregistre la valeur moyenne la plus élevée, 36,8 $\pm 9,63 \%$, tandis que le sol gravillonnaire 
enregistre la plus faible valeur, 7,8 \pm 3,65\% Le sol sableux induit donc les meilleurs taux de jeunes plants susceptibles de survivre et de se développer en plants de bonne qualité. Pour la valeur culturale, le sol sableur enregistre également la plus forte valeur $(42,07 \pm 5,99 \%)$ par rapport au sol argileux, 12,82 $\pm 5,22 \%$, et au sol gravillonnaire, 4,95 $\pm 1,31$. Le sol sableux donc permet d'obtenir un semis pas trop clair, ni dru, avec une quantité moins importante de semence que prévue.

\subsection{Influence de l'intenté de} l'ensoleillement sur la croissance des plants issus des graines et des stumps : Le tableau 4 récapitule l'évolution des paramètres observés (hauteur des plants et diamètre des tiges) et la comparaison des moyennes entre les milieux et entres les deux types de matériel végétal, pour ces différents paramètres de croissance. Durant le premier mois d'essai, le milieu ombragé induit une croissance en hauteur des plants issus des graines relativement supérieure, 10,23 \pm 2,06 $\mathrm{cm}$, au milieu ouvert, 9,94 $\pm 1,82 \mathrm{~cm}$. Au bout de cinq mois de croissance, le constat est le même, pour des valeurs de 28,07 $\pm 7,2 \mathrm{~cm}$, dans le milieu ombragé, contre $25,21 \pm 7,26 \mathrm{~cm}$, dans le milieu ouvert. Le constat est le même pour la vitesse de croissance dont les valeurs sont de $3,57 \pm 1,47 \mathrm{~cm} / \mathrm{mois}$, dans le milieu ombragé, contre $3,05 \pm 1,88 \mathrm{~cm} / \mathrm{mois}$, dans le milieu ouvert. En ce qui concerne les stumps, les plants atteignent une taille de 10,36 $\pm 2,49 \mathrm{~cm}$ en milieu ombragé et, seulement 5,34 \pm 1,69 cm en milieu ouvert. Au bout des 5 mois de croissance, la hauteur reste supérieure en milieu ombragé, bien qu'aucune différence significative ne soit été notée entre les valeurs moyennes. En milieu ombragé, les plants issus des stumps, ont une croissance plus rapide, que ceux issus de graines sur la période de 5 mois. Dans le milieu ouvert, les plants issus des stumps ont enregistrés une croissance relativement plus élevée, $28,28 \pm 4,7$ $\mathrm{cm}$, bien que on observe une absence de différence statistique significative entre les moyennes de la hauteur de croissance. Pour la croissance en diamètre, le milieu ouvert induit une meilleure croissance des plants issus des graines $(0,48 \pm 1,07 \mathrm{~cm})$ sur la période de cinq mois d'observation. Mais aucun effet statistique significatif n'a été noté entre les valeurs moyennes du paramètre de croissance. Les résultats sont identiques pour les plants issus des stumps, dont les valeurs sont de 0,68 $\pm 0,64 \mathrm{~cm}$, pour le milieu ouvert, et de $0,62 \pm 0,75 \mathrm{~cm}$, pour le milieu ombragé. L'analyse comparative de la croissance en diamètre (Figure 5) des deux types de matériel végétal montre que les plants issus des stumps développent une croissance supérieure, quel que soit le milieu considéré, sur la période d'essai. On note un effet statistique assez significatif entre les valeurs moyennes de ce paramètre de croissance, dans le milieu ombragé, tout comme dans le milieu ouvert. 


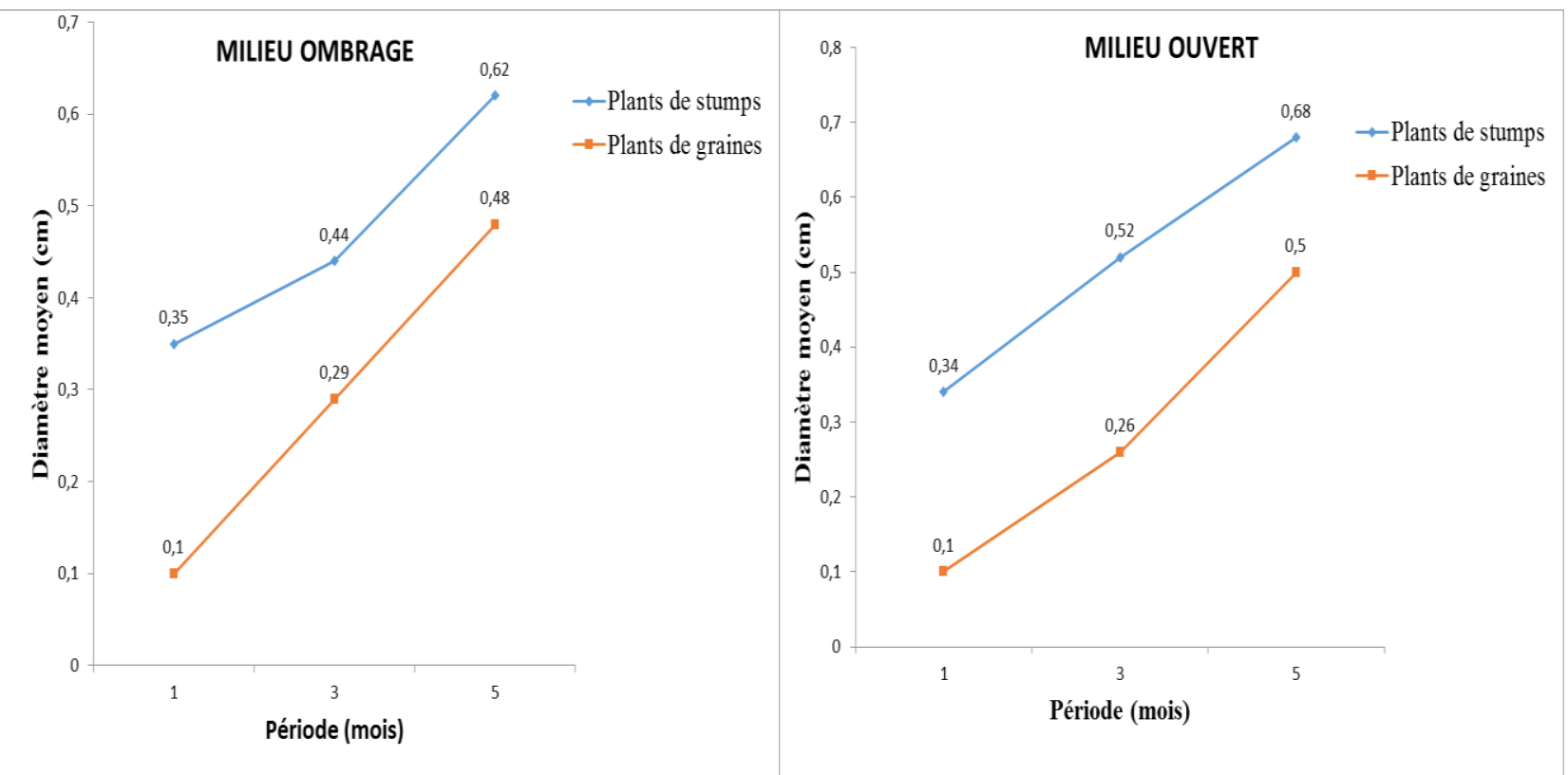

Figure 5 : Croissance en diamètre des plants issues des graines et des stumps de Khaya senegalensis, en milieu ombragé et en milieu ouvert

\section{DISCUSSION}

\subsection{Influence de l'intensité} d'ensoleillement sur les paramètres de germination des semences : La forte intensité (milieu ouvert), qui réduit l'humidité du sol, ne semble pas ralentir les réactions métaboliques de l'embryon de la graine et de facto la sortie rapide de la radicule chez l'espèce. Des observations similaires ont été faites par Kouadio et al., (2014) sur les semences d'Entandrophragma angolense et Guibourtia ehie. Par contre, les taux de germination élevés, en milieu ombragé, montrent bien que l'ombrage, qui crée un effet de microclimat, stimule la levée de la dormance des semences de $K$. senegalensis et caractérise l'habitat optimal (forêt galerie) de cette espèce. Les semences sont donc moins exigeantes en lumière pour leur germination. Selon Kouadio et al. (2014), chez une telle espèce, les phytochromes localisés dans ses semences, ont besoin d'intensité lumineuse modérée pour faciliter la sortie des radicules. En plus, l'ombrage permet de limiter l'évaporation d'eau du sol et de conserver ainsi l'humidité pendant longtemps ; ce qui réduit ainsi la température du milieu (Natta et al., 2013). Considérant l'énergie germinative, paramètre qui renseigne un peu sur la survie et la mortalité des plantules après le stade de germination, le milieu ombragé influence mieux ce paramètre. Plus de $50 \%$ des graines viables germent pour des périodes énergétiques respectives de 16 à 20 jours. Bationo (1994), a obtenu un taux similaire avec des semences de Guiera senegalensis pour une période énergétique de 17 jours. Cette valeur de l'énergie germinative, obtenue en milieu ombragé, est un bon indicateur qui pourrait encourager pour la production de plants de $K$. senegalensis en pépinière, afin d'assurer son reboisement dans les milieux dégradés.

5.2. Influence du type de sol cultural sur les paramètres de germination des semences : L'analyse de l'effet des trois sols sur les qualités germinatives, a révélé que le sol sableux induit les meilleurs résultats des paramètres de germination, chez K. senegalensis. Les paramètres de germination, considérés, sont intermédiaires sur sol argileux et plus faibles sur sol gravillonnaire. Nos résultats corroborent ceux de Onana (2009) qui a observé de meilleurs paramètres de germination de Pericopsis elata sur sol sableux. Le sable permet un bon contact entre les semences et la source d'humidité 
(Willan, 1992). Cependant, des résultats contraires ont été obtenus par Houehounha et al. (2009) avec Daniellia oliveri sur des sols de textures identiques à celles de notre étude. De même, Sanou (2014) a obtenu de faibles taux de germination de Sterculia setigera sur sol sableux, au Burkina Faso. Ces différences de résultats peuvent être liées aux facteurs physicochimiques des sols mais aussi à la germination hypogée des graines de K. senegalensis caractérisée par l'émergence de la radicule qu'après que la radicule ait atteint une certaine profondeur dans le sol. La structure compacte des sols argileux et gravillonnaire peut donc freiner la croissance de la radicule et retarder ainsi l'émergence de la tigelle.

5.3. Influence de la profondeur de semis sur les paramètres de germination des semences: Les résultats des essais de germination traduisent une décroissance régulière des valeurs des paramètres de germination des semences de $K$. senegalensis avec un seuil de tolérance qui se situe à $3 \mathrm{~cm}$ de profondeur. Au-delà de cette profondeur, les paramètres de germination sont influencés significativement par la résistance mécanique et par l'épaisseur de la couche supérieure de terre que doit traverser les tigelles issues des graines lors de leur croissance (Gérard, 1980). Selon cet auteur, la poussée exercée par la plantule est proportionnelle à la section de l'hypocotyle (en général liée à la dimension de la graine) et est accrue en bonne conditions d'alimentation hydrique (turgescence). Ce qui, par ailleurs, permet de réduire la cohésion de la terre. Bowers et Hayden (1972) ont montré, dans leur étude sur le haricot, que pour les plantes à tigelle

\section{CONCLUSION}

La présente étude a permis d'apprécier l'effet des facteurs abiotiques sur les qualités germinatives des graines et sur la croissance juvénile des plants issus de deux techniques de multiplication artificielle de l'espèce $K$. senegalensis. Pour les paramètres de germination considérés, le milieu ombragé a présenté les moyennes les plus élevées. Pour les substrats, le sol sableux s'est révélé être plus propice à la gémination de masse hypocotylée (germination épigée), la position de la graine influençait le pourcentage de germination.

5.4. Influence de l'intensité de la lumière sur la dynamique de croissance des plants : Le milieu ombragé s'est montré favorable à la croissance en hauteur des plants par rapport au milieu ouvert. Les fortes intensités lumineuses serraient donc néfastes pour le développement vertical des jeunes plants de l'espèce. Kouadio et al. (2013) ont signalé aussi l'effet bénéfique du milieu ombragé sur la croissance en hauteur de plants produits en pépinière. Cette forte croissance, serait due au phénomène de l'étiolement par lequel la plantule, organisme hétérotrophe, utilise toutes ses ressources pour assurer la croissance dans la tige à la recherche de la lumière (Prat, 2008), Ce phénomène est dû au fait qu'en absence de lumière, l'auxine est fortement active et provoque la croissance exacerbée de la tige (Vitre, 2012). Dans un même environnement, les différences significatives de croissance en hauteur et en diamètre des deux types de matériel végétal s'expliquent par le fait que les stumps, issus de plants d'âge d'environ un an, ont emmagasiné des réserves nutritives qui assurent la reprise et la croissance rapide des repousses. En ce qui concerne la croissance en diamètre, les plants, quel que soit leur origine, ont un meilleur développement en milieu ouvert. Nos résultats sont contraires à ceux de Kouadio et al. (2013) qui ont noté que l'ensoleillement modéré stimulait la croissance en diamètre de Guibourtia ebie. C'est donc à juste titre que M'Sadak et al. (2013) affirment que le diamètre au collet est une variable qui intègre la réponse morphologique aux facteurs environnementaux.

des semences de l'espèce. Par ailleurs, l'essai de semis des semences à différentes niveaux de profondeur a permis de noter que le potentiel de germination reste meilleur pour des semis allant jusqu'à $5 \mathrm{~cm}$ de profondeur. Le suivi des paramètres de croissance, notamment la hauteur, a montré que l'espèce a un meilleur potentiel de croissance en milieu ombragé pour les deux techniques de multiplication. Dans les 
conditions de l'essai, les plants de $K$. senegalensis issus du repiquage de stumps ont donné la meilleure performance de croissance tant en hauteur, qu'en diamètre. A la suite de cette étude qui constitue un complément d'informations à la

\section{REMERCIEMENTS}

Nous adressons nos remerciements aux Agents du Cantonnement des Eaux et Forêts du sylviculture de l'espèce, il serait utile d'explorer d'autres voies de multiplication, notamment celles du bouturage et du marcottage pour l'obtention d'un matériel végétal en grande quantité et à très court terme.

Département de Kouto pour leurs contributions à la réalisation de cette étude.

\section{REFERENCES BIBLIOGRAPHIQUES}

Adjahossou SGC, Yaoitcha A., Houehanou DT, Gouwakinnou GN, Sode AI, Houinato M., \& Sinsin B.-2018. Favorable conservation habitats of Khaya senegalensis in Benin. Data sheets. National Library, Benin, 12 p.

Bationo BA, 1994.- Study of the agroforestry potentialities of the multiplication and uses of Guiera senegalensis JFGMEL. Rural Development Engineer thesis, University of Ouagadougou, Burkina Faso, 74 p.

Beaudou A .G. \& Sayol R., 1980.- Pedological study of the Boundiali-Korhogo region (Ivory Coast). ORSTOM Paris, explanatory note $\mathrm{n}^{\circ} 84,35 \mathrm{p}$.

Bowers SA, \& Hayden CW, 1972. - Influence of seed orientation on bean seedling emergence. Agronomy Journal, 64: 736738. https://doi.org/10.2134/agronj 1972.00021962006400060008x

CIRAD, 1988. -Khaya senegalensis (Desr.) A. Juss. Tropical Woods and Forests, 218: 43 56.

Douma S., Adamou MM, Aboubacar K., Alleidi I. \& Boubacar AN, 2019. Effect of the irrigation regime on the germination and growth in the nursery of Parkia biglobosa (Jacq.) G. Don. Journal of Animal \& Plant Sciences, 40 (1): 65736583.

Eyog MO, 1987.- Monographic study of Cailcédrat; Khaya senegalensis (Desr) A. Juss. (Meliaceae). Nkolbisson Forest Research Center, Maroua Branch, Cameroon. 26 p.
FAO, 2005. -State of the World's Forests available at http://www.fao.org/3/ay5574e.pdf (page consulted on March 11, 2020).

Gérard CJ, 1980.- Emergence force by cotton seedlings. Agronomy Journal, 72: 473 476. https://doi.org/10.2134/agronj 1980.00021962007200030016x

Houehounha R., Avohou HT, Sinsin B., \& Tandiepkon AM 2009. Approaches to artificial regeneration of Daniella oliveri (Rolfe) Hutchison and Dalziel. International Journal of Biological and Chemical Sciences, 3 (1): 7-19. https://doi.org/10.4314/ijbcs.v3i1.427 30

Inoussa TM, Ismaila TI, Gbègbo MC, \& Sinsin B., 2013.- Floristic structure and composition of the dense dry forests of the Kouffé Mountains. Journal of Applied Biosciences, 64: 4787-4796. https://doi.org/10.4314/jab.v64i1.8846 7

Kolawole OT, Kolawole SO, Ayankunle AA \& Olaniran OI 2012. - Anti-hyperglycemic effect of Khaya senegalensis Stem bark aqueous extract in Wistar Rats. European Journal Medicinal Plants, 2 (1): 66-73. https://doi.org/10.9734/EJMP/2012/ 934

Kouadio K., Dibi NH, Bomisso L. \& Ettien KBR, 2013.- Effect of sunshine intensity on the initial growth in the nursery of Guibourtia ehie (A. Chev.) Leonard (Caesalpiniaceae), a commonly exploited and threatened species extinction, in the 
Forest Management Unit of Bossématié (Ivory Coast). International Journal of Biological and Chemical Sciences, 7 (6): 2292-2300. https://doi.org/10.4314/ ijbcs.v7i6.10

Kouadio K., Koné M. \& Soro D., 2014.Influence of sunshine on the germination of four endangered forest species in Ivorian forests. Journal of Animal \& Plant Sciences, 23 (1): 35293538.

M'sadak Y., Elouaer M. A, \& Dhahri M. 2012. Comparative growth of okra plants in field culture according to direct sowing and transplanting modes. Algerian Journal of Arid Environment, 2 (2): 6270.

Natta AK, Yedomonhan H., Zoumarou-wallis N., Houndehin J., Ewedje EBK \& Glèlé Kakai RL 2011. Typology and structure of natural populations of Pentadesma butyracea in the Sudano-Guinean area of Benin. Annals of Agronomic Sciences, 15 (2): 137-152.

Natta AK, Zoumarou-Wallis N., Akossou AYJ, \& Houndehin J. 2013. - Effects of water and light on the germination of Pentadesma butyracea Sabine seeds in central Benin. Annals of the University of Parakou, Series "Natural Sciences and Agronomy, 2 (2): 9-15

Olivier M., Zerbo P., Boussim JI \& Guinko S., 2012. Riparian forest plants for traditional use by traditional health practitioners and Dozo Sénoufo hunters in Burkina Faso. International Journal of Biological and Chemical Sciences, 6 (5): 2170-2191. https://doi.org/10.4314/ijbcs.v6i5.24
Onana MH, 2009.- Silviculture and natural regeneration of Pericopsis elata (Harms) Var. Meeuwen in plantation: the case of the eastern and southern regions. Water, Forest and Hunting Engineer thesis, University of Dschang, Cameroon, 88 p. Prat, R., 2008. Plant biology: Growth and Development, 2nd Edition 256 p.

Sanogo S., Sacandé M., Damme PV \& NDiaye I., 2013.- Characterization, germination and conservation of seeds of Carapa procera DC. (Meliaceae), a useful species in human and animal health. Biotechnology, Agronomy, Society and Environment, 17 (2): 321-331.

Sanou ZHR, 2014.- Contribution to the domestication of Sterculia setigera del. in Burkina Faso: determination of seed germination conditions and evaluation of seedling growth parameters. Master's thesis, Polytechnic University of BoboDioulasso, Burkina Faso, 50p.

Silué PA, 2018.- Studies of flora, vegetation dynamics and germination test of three species commonly exploited from the classified forests of Palé and Pouniakélé, in the region of Bagoué (North-West of Côte d 'Ivory). Doctoral thesis, UFR Biosciences., Félix Houphouët Boigny University, Ivory Coast, 256 p.

Vitre A., 2012.- Management of greenhouse work for tomato cultivation, $18 \mathrm{p}$. www.agrieseau.qc.ca/legumesdeserre. Site consulted on February 12, 2020.

Willan RL, 1992.- Guide to handling forest seeds (in the particular case of tropical regions). FAO Forestry Study, 20/2. Rome: FAO., 443 p.. 
Tableau 1 : Valeurs moyennes des principaux paramètres de germination des différents types de semences de Khaya senegalensis, en milieu ombragé et en milieu ouvert

\begin{tabular}{|c|c|c|c|c|c|}
\hline Paramètres & $\begin{array}{c}\text { Semences traitées à } \\
\text { l'eau sur milieu } \\
\text { ombragé }\end{array}$ & $\begin{array}{c}\text { Semences traitées à } \\
\text { l'eau sur milieu } \\
\text { ouvert }\end{array}$ & $\begin{array}{l}\text { Semences non traitées } \\
\text { sur milieu ombragé }\end{array}$ & $\begin{array}{c}\text { Semences non } \\
\text { traitées sur milieu } \\
\text { ouvert } \\
\end{array}$ & Statistique du test \\
\hline TL (jours) & $13,00^{\mathrm{a}} \pm 1,00$ & $12,60^{\mathrm{a}} \pm 0,54$ & $14,40^{\mathrm{b}} \pm 0,89$ & $14,60^{\mathrm{b}} \pm 0,55$ & $\begin{array}{c}\chi^{2}=11,67 ; p= \\
0,002\end{array}$ \\
\hline TG (\%) & $86,40 \pm 3,91^{\mathrm{a}}$ & $68,00 \pm 6,12^{b}$ & $81,80 \pm 10,26^{a}$ & $64,60 \pm 4,56^{b}$ & $\begin{array}{c}F=12,42 ; p= \\
0,0002\end{array}$ \\
\hline EG (\%) & $51,80 \pm 9,90^{a}$ & $37,00 \pm 15,70^{\mathrm{a}}$ & $53,00 \pm 9,43^{a}$ & $44,40 \pm 18,38^{a}$ & $F=1,42 ; p=0,27$ \\
\hline $\mathrm{VC}(\%)$ & $80,95 \pm 5,99^{a}$ & $57,01 \pm 9,40^{\mathrm{bc}}$ & $73,45 \pm 13,96^{\mathrm{ab}}$ & $51,98 \pm 8,96^{c}$ & $F=9,68 ; p=0,001$ \\
\hline
\end{tabular}

$\mathrm{TL}=$ Temps de latence $; \mathrm{TG}=$ Taux de germination $; \mathrm{EG}=$ Energie germinative $; \mathrm{VC}=$ Valeur culturale.

NB : sur chaque ligne il n'y a pas de différences significatives entre les moyennes suivies d'une même lettre au seuil de $\alpha=0,05$

Tableau 2 : Valeurs moyennes des principaux paramètres de germination des semences de Khaya senegalensis en fonction de la profondeur de semis

\begin{tabular}{|c|c|c|c|c|c|}
\hline Paramètres & Groupe 1 & Groupe 2 & Groupe 3 & Groupe 4 & Statistique du test \\
\hline TL (jours) & $26,40 \pm 0,55^{\mathrm{a}}$ & $25,60 \pm 0,55^{\mathrm{a}}$ & $15,80 \pm 1,09^{b}$ & $13,06 \pm 0,59 c$ & $\chi^{2}=7,81 ; p<0,0001$ \\
\hline TG (\%) & $17,40 \pm 1,14^{a}$ & $38,40 \pm 1,14^{\mathrm{ab}}$ & $67,00 \pm 7,78^{\mathrm{b}}$ & $83,06 \pm 4,79 c$ & $\chi^{2}=7,81 ; p<0,0001$ \\
\hline EG (\%) & $11,00 \pm 1,0^{\mathrm{a}}$ & $14,20 \pm 2,68^{\mathrm{ab}}$ & $33,20 \pm 10,83^{\mathrm{ab}}$ & $52,87 \pm 7,62^{c}$ & $\chi^{2}=7,81 ; p<0,0001$ \\
\hline VC (\%) & $3,64 \pm 0,48^{a}$ & $15,06 \pm 0,94 \mathrm{ab}$ & $54,81 \pm 8,98^{\mathrm{ab}}$ & $72,74 \pm 7,14 c$ & $\chi^{2}=7,81 ; p<0,0001$ \\
\hline
\end{tabular}

$\mathrm{TL}=$ Temps de latence $; \mathrm{TG}=$ Taux de germination $; \mathrm{EG}=$ Energie germinative $; \mathrm{VC}=$ Valeur culturale

NB : sur chaque ligne il n'y a pas de différences significatives entre les moyennes suivies d'une même lettre au seuil de $\alpha=0,05$ 
Tableau 3 : Valeurs moyennes des principaux paramètres de germination des semences de Khaya senegalensis par types de sols

\begin{tabular}{l|c|c|c|c}
\hline Paramètres & Sol sableux & Sol argileux & Sol gravillonnaire & Statistique du test \\
\hline TL (jours) & $12,6 \pm 0,55^{\mathrm{a}}$ & $12,6 \pm 0,55^{\mathrm{a}}$ & $15,6 \pm 3,58^{\mathrm{a}}$ & $\chi^{2}=5 ; p=0,08$ \\
\hline TG (\%) & $61,8 \pm 4,60^{\mathrm{a}}$ & $30,8 \pm 6,1^{\mathrm{b}}$ & $16 \pm 3,53^{\mathrm{c}}$ & $F=3,7 ; p<0,0001$ \\
\hline EG (\%) & $36,8 \pm 9,63^{\mathrm{a}}$ & $23,2 \pm 11,78^{\mathrm{b}}$ & $7,4 \pm 3,65^{\mathrm{c}}$ & $\chi^{2}=10,88 ; p=0,0002$ \\
\hline VC (\%) & $42,07 \pm 5,99^{\mathrm{a}}$ & $12,82 \pm 5,22^{\mathrm{b}}$ & $4,95 \pm 1,31^{\mathrm{c}}$ & $\chi^{2}=12,5 ; p<0,0001$ \\
\hline
\end{tabular}

$\mathrm{TL}=$ Temps de latence $; \mathrm{TG}=$ Taux de germination $; \mathrm{EG}=$ Energie germinative $; \mathrm{VC}=$ Valeur culturale

NB : sur chaque ligne il n'y a pas de différences significatives entre les moyennes suivies d'une même lettre au seuil de $\alpha=0,05$

Tableau 4: Valeurs moyennes des paramètres de croissance des types de plants de Khaya senegalensis en fonction du milieu (ombragé, ouvert)

\begin{tabular}{c|c|c|c|c}
\hline Paramètres & $\begin{array}{c}\text { Origines des } \\
\text { plants }\end{array}$ & Milieu ombragé & Milieu ouvert & Statistique du test \\
\hline \multirow{2}{*}{$\begin{array}{c}\text { Hauteur totale moyenne } \\
\text { initiale }(\mathbf{c m})\end{array}$} & Graines & $10,23 \pm 2,06$ & $9,94 \pm 1,82$ & $t=0,38 ; p=0,7$ \\
\cline { 2 - 5 } & Stumps & $10,36 \pm 2,49$ & $5,34 \pm 1,69$ & $t=5,54 ; p<0,0001$ \\
\hline \multirow{2}{*}{$\begin{array}{c}\text { Hauteur totale moyenne finale } \\
(\mathbf{c m})\end{array}$} & Graines & $28,07 \pm 7,25$ & $25,21 \pm 7,26$ & $t=1,02 ; p=0,31$ \\
\cline { 2 - 5 } & Stumps & $35,32 \pm 5,48$ & $28,28 \pm 4,79$ & $t=3,21 ; p=0,004$ \\
\hline $\begin{array}{c}\text { Vitesse de croissance en } \\
\text { hauteur moyenne (cm/mois) }\end{array}$ & Graines & $3,57 \pm 1,47$ & $3,05 \pm 1,58$ & $t=0,37 ; p=0,39$ \\
\cline { 2 - 5 } Diamètre moyen au collet (cm) & Stumps & $4,99 \pm 1,31$ & $4,59 \pm 0,81$ & $t=0,87 ; p=0,4$ \\
\cline { 2 - 5 } & Graines & $0,48 \pm 1,07$ & $0,5 \pm 0,88$ & $U=77 ; p=0,5$ \\
\hline
\end{tabular}

